\title{
Early endoscopic transpapillary drainage through the minor papilla in the treatment of acute pancreatitis
}

\author{
Yafei Yang $^{1,2 \#}$, Weijie Yao ${ }^{1 \#}$, Zhu Lan ${ }^{1,2}$, Chengsi Zhao ${ }^{1,2}$, Bo Peng ${ }^{1,2}$, Tongtong Dong ${ }^{1,2}$, Jinping Shi ${ }^{1,2}$, \\ Zuozheng Wang ${ }^{1}$
}

${ }^{1}$ Department of Hepatobiliary Surgery, General Hospital of Ningxia Medical University, Yinchuan, China; ${ }^{2}$ School of Clinical Medicine, Ningxia Medical University, Yinchuan, China

Contributions: (I) Conception and design: W Yao, Y Yang; (II) Administrative support: None; (III) Provision of study materials or patients: None; (IV) Collection and assembly of data: Z Lan, C Zhao, B Peng; (V) Data analysis and interpretation: T Dong, J Shi; (VI) Manuscript writing: All authors; (VII) Final approval of manuscript: All authors.

\#These authors contributed equally to this work.

Correspondence to: Zuozheng Wang. Department of Hepatobiliary Surgery, General Hospital of Ningxia Medical University, No. 804 Shengli South Street, Xingqing District, Yinchuan 710004, China. Email: wzz13895009292@163.com, wangzuozheng2008@sina.com.

\begin{abstract}
Background: To explore the feasibility, safety, and efficacy of endoscopic transpapillary drainage through the minor papilla in the treatment of acute pancreatitis (AP).

Methods: We retrospectively evaluated the safety and efficacy of endoscopic transpapillary drainage via the minor papilla among AP patients who were treated in our hospital from September 2018 to March 2020.

Results: The present study included 18 patients (12 males and 6 females). All patients successfully received endoscopic transpapillary drainage via the minor papilla and were discharged upon recovery. No patient died, received ICU treatment, or had endoscopic operation-related complications. Two patients $(11.11 \%)$ received additional abdominal paracentesis due to local complications. Fifteen patients (83.33\%) resumed oral feeding within 3 days. The postoperative 24-hour leukocyte level, APACHE II score, serum amylase level, and lipase level significantly decreased compared with those at admission. The median hospitalization stay was 5 (3.75-9) days. The median hospitalization cost was 25,123.82 (22,942.50-43,874.68) RMB. The patients were followed up at 6-24 months, during which 4 patients $(22.22 \%)$ had recurrence. Two patients had recurrence after pancreatic duct removal and other 2 patients in the period of carrying ducts.

Conclusions: Early endoscopic transpapillary drainage via the minor papilla in cases of difficult cannulation or stenting via the major papilla is safe and effective in the treatment of AP, and is worthy of further popularization.
\end{abstract}

Keywords: Acute pancreatitis (AP); minor duodenal papilla; pancreatic stents; endoscopic treatment

Submitted Dec 22, 2021. Accepted for publication Feb 18, 2022.

doi: $10.21037 /$ gs-22-38

View this article at: https://dx.doi.org/10.21037/gs-22-38

\section{Introduction}

Acute pancreatitis (AP) is a common clinical emergency requiring hospital admission. It is characterized by significant morbidity and a high case fatality rate (1). The incidence of AP has gradually increased in China in the past few decades (2). Currently, conservative treatment for AP remains an important strategy, including fluid resuscitation, antibiotic usage, early enteral nutrition, specific drugs, and combined treatment with traditional Chinese medicine (3). Some severe AP patients may develop complications of acute peripancreatic fluid accumulation, pancreatic pseudocyst, acute necrotic collection, and walled-off necrosis, among others, requiring surgical intervention (4,5). However, treatment is not effective in a portion of patients who have to endure both a protracted disease course and extra financial burden. Endoscopic therapy in AP has been shown 
to play important roles in AP treatment due to its minimal invasiveness and obvious benefits $(6,7)$.

Among the current endoscopic techniques in AP treatment, endoscopic transpapillary pancreatic drainage (ETPD) has received much attention due to the importance of releasing pancreatic duct obstructions and relieving hypertension in AP treatment. Harvey et al. established AP cat models induced by bile reflux, alcohol, and pancreatic duct obstruction, and the 3 types of AP all showed pancreatic duct hypertension (8). Moreover, 31-44\% of patients with acute necrotizing pancreatitis were shown to have rupture of the pancreatic duct (9-11), in which pancreatic duct hypertension aggravates peripancreatic fluid exudation and inflammatory reactions (12). Thus, pancreatitis is closely associated with pancreatic hypertension regardless of its etiology. To date, there have been several studies reporting successful application of pancreatic duct decompression in the treatment of AP (13-16), and ETPD is a widely used technique for pancreatic duct decompression.

During the process of ETPD, physicians may find it difficult to perform cannulation via the major papilla, for example, the guidewire passes through the pancreas head but does not reach the pancreas tail. At the same time, the minor papilla presents swelling and protrusion, suggesting minor pancreatic duct hypertension. The minor pancreatic duct is the major draining duct for the dorsal pancreas during the embryonic phase, and the minor pancreatic duct completely or partially fuses with the major pancreatic duct at the end of embryonic development (17). However, pancreatic duct development may have variation in a small population (17), thus leading to pancreatic divisum, pancreatic duct dysplasia, or deformation. Generally, the minor pancreatic duct is located above the pancreatic head and enters the duodenum at the minor duodenal papilla (18). It may be involved in the drainage of pancreatic secretions, and the degree of involvement depends on pancreatic duct variation. Swelling of the minor duodenal papilla suggests not only obstruction of the minor pancreatic duct, but also minor pancreatic duct variation or pancreatic duct disruption. Therefore, in these cases, we managed to perform transpapillary drainage through the minor papilla to drain the pancreatic fluid. To date, our hospital has accumulated experience in transpapillary drainage through the minor papilla in AP treatment.

At present, much progress has been made on the application of endoscopic therapy in the treatment of biliary and pancreatic diseases, especially AP with cholangitis or biliary duct obstruction (6). However, there are few reports regarding early endoscopic transpapillary drainage, especially through the minor papilla, in the treatment of AP. Therefore, in this study, we retrospectively reviewed the treatment of AP patients who received early ETPD in our hospital whose drainage was managed via the minor papilla, aiming to evaluate the efficacy and safety of this treatment strategy and technique for AP. We present the following article in accordance with the STROBE reporting checklist (available at https://gs.amegroups.com/article/ view/10.21037/gs-22-38/rc).

\section{Methods}

\section{Patient inclusion}

We retrospectively reviewed the electronic medical records of patients who received ETPD for AP and drainage of the minor papilla performed due to difficult cannulation in the major duodenal papilla from September 2018 to March 2020 in our hospital. The data of clinical characteristics, adverse events, clinical course, and outcomes. Diagnosis criteria, rating criteria, and local complication definitions all followed the Classification of Acute Pancreatitis 2012 (19). CT classification and rating followed the Balthazar CT evaluation system (20). All patients provided written informed consent. All procedures performed in this study involving human participants were in accordance with the Declaration of Helsinki (as revised in 2013). The study was approved by institutional ethics board of General Hospital of Ningxia Medical University (No. KYLL-2022-1104).

\section{Technique}

Therapeutic duodenoscope (Olympus TJF-240, 260), papillotome (Olympus KD-V411M-720), guidewire (COOK ACRO-35-450), and a 5- or 7-Fr and 4-12 cm-long pancreatic stent $(\mathrm{COOK})$ were used. Patients were fasted for more than 6 hours before the operation. Anisodamine hydrobromide $0.6 \mathrm{mg}$ was intramuscularly injected and dezocine $5 \mathrm{mg}$ was intravenously injected. After 3 minutes, propofol $2 \mathrm{mg} / \mathrm{kg}$ was intravenously injected. After anesthesia induction, a micropump was connected to the patient. Then, propofol $2-5 \mathrm{mg} / \mathrm{kg} / \mathrm{h}$ and dexmedetomidine hydrochloride $0.05-0.1 \mu \mathrm{g} / \mathrm{kg} / \mathrm{min}$ were intravenously injected for continuous anesthesia. Patients underwent surgery when the eyelash reflex disappeared. Duodenoscopy accessed the esophagus and gastric lumen and reached the inner side of the duodenum. Major papilla cannulation was performed. In cases with difficult cannulation via the major papilla (for example, the guidewire could not reach the 
Table 1 Characteristics of included AP patients

\begin{tabular}{lc}
\hline Characteristics & Included patients $(\mathrm{n}=18)$ \\
\hline Gender (\%) & $12(66.67)$ \\
Male & $6(33.33)$ \\
Female & $20-77$ years $(40$ years) \\
Age, range (mean) & $4(22.22)$ \\
Recurrent AP (\%) & $7(38.89)$ \\
Combined etiology (\%) & $4(22.22)$ \\
Cholelithiasis & $1(5.55)$ \\
Hyperlipidemia & $1-4$ days $(2$ days) \\
Alcohol consumption & \\
Duration of symptoms (median) & $11(61.11)$ \\
CTSI score & $5(27.78)$ \\
$0-3$ & $2(11.11)$ \\
$4-6$ &
\end{tabular}

CTSI score, CT severity index; AP, acute pancreatitis.

tail of the pancreas), a shorter stent was used for drainage if allowed. If minor papilla swelling was found, then cannulation and stent insertion was performed through the minor papilla. Sphincterotomy and bile duct management were performed when necessary.

\section{Postoperative management}

All patients were treated with regular fasting, antacid, and rehydration therapy. In case of elevated inflammatory factor levels, antibiotics were given. Oral feeding was resumed when abdominal pain and distention were obviously relieved [NRS (Digital pain score) pain score $\leq 3$ ] and blood amylase $<300$ U/L. Magnetic resonance cholangiopancreatography (MRCP) was performed among patients to identify minor pancreatic variations. Patients who had no AP and operation-related complications, had normal hemogram parameters as indicated by routine blood tests, and could resume oral feeding were discharged from hospital.

\section{Follow up}

The patients were followed up in their regular outpatient clinic visits at our department after the operation, when clinical variables, imaging results, and postoperative outcomes were evaluated. Before removal of pancreatic ducts, routine blood and biochemical tests were performed at 2-week intervals, and abdominal enhanced CT examinations were performed at 1 month intervals. Evaluation for stent removal was performed at 2 months after discharge. When there was no abdominal discomfort and peripancreatic exudation was absorbed completely, pancreatic ducts could be removed. For patients with stents who had recurrence, stent obstruction was considered, and ducts needed to be replaced. If AP recurrence developed after stent removal, stent replacement could be performed. After stent removal, patients were followed up through telephone interview regarding diet, abdominal discomfort, recurrence, weight. Follow up ended after 24 months for all patients if there was no abnormal situation.

\section{Clinical variables}

The clinical variables collected included oral feeding time, hospital stay, pre- and postoperative 24-hour leukocyte levels, serum lipase levels, serum amylase levels, APACHE II score, mortality rate, AP-related complication rate, endoscopic operation-related complication rate, additional surgical intervention due to AP-related complications, hospitalization cost, AP recurrence during follow up.

\section{Statistical analysis}

Statistical analyses were performed with SPSS 22.0. Continuous variables were analyzed for normal distribution and variance homogeneity and were shown as mean \pm standard deviation (SD). Categorical variables were shown as percentage. Comparisons of continuous variables between 2 groups were performed with the following tests: Dunnett's test (for normal distribution), Mann-Whitney U test (for skewed distribution), Brown-Forsythe or Welch's test (for heterogeneity of variance). The chi-square test was used to compare categorical variables between 2 groups. Values of $\mathrm{P}<0.05$ were statistically significant.

\section{Results}

\section{Characteristics of the included patients}

A total of 18 AP patients who received endoscopic transpapillary drainage through the minor papilla (12 men, $66.67 \%$ ) in our hospital were included in the present study. The characteristics of the patients are summarized in Table 1 . The median age was 40 years (range, 20-77 years). 

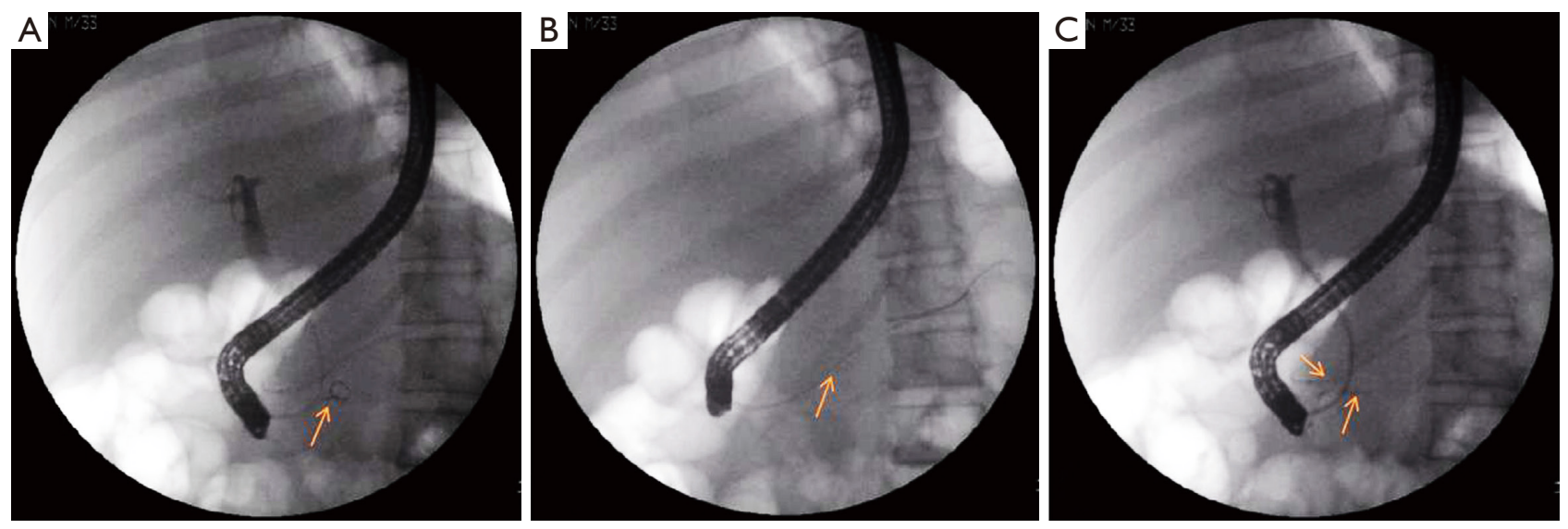

Figure 1 ERCP angiography results. (A) The guidewire could not reach the pancreatic tail despite multiple cannulation via the major pancreatic duct; (B) the guidewire successfully reached the pancreatic tail in cannulation via the minor pancreatic duct; (C) two stents were placed in the major and minor pancreatic ducts. The stent placed via the minor papilla accessed the minor pancreatic duct and reached the pancreatic tail. The stent placed via the major papilla was located at the pancreatic head and neck. The arrow indicates the location of pancreatic duct stent.
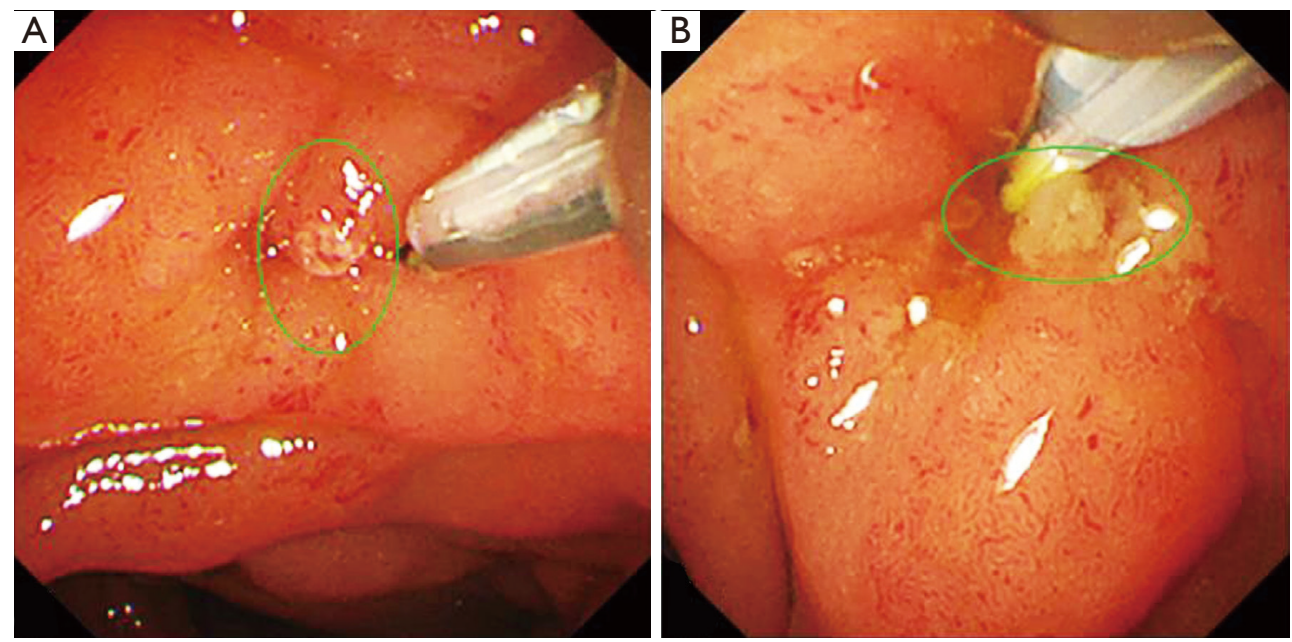

Figure 2 Schematic diagram of direct vision under duodenoscopy. (A) The protruding minor papilla was obvious under duodenoscopy; (B) the obstruction mass flew out from pancreatic ducts upon suction after cannulation. The green circles indicate the treatment site and its changes.

Four patients $(22.22 \%)$ had recurrent pancreatitis. Seven patients $(38.89 \%)$ had combined etiology of cholelithiasis, 4 patients $(22.22 \%)$ had combined hyperlipidemia, and 1 patient $(5.55 \%)$ had alcohol consumption. The median duration of symptoms was 2 days. Eleven patients (61.11\%) had a CTSI score of $0-3,5$ patients $(27.78 \%)$ had a CTSI score of $4-6$, and 2 patients (11.11\%) had a score of $7-10$.

\section{Operation process and outcomes}

Among the 18 included patients, both the major papilla and minor papilla were placed with stents, thus the pancreatic ducts were drained via 2 papillae (Figure 1). The median operation time was 29 [22-38] minutes. During the operations, gastroduodenal erosion bleeding was found in most patients. When suction was performed along the papillotome after cannulation, we observed that an obstruction mass flew out from pancreatic ducts in some patients (Figure 2).

\section{Short-term outcomes}

The short-term outcomes are summarized in Tables 2-4. 
Table 2 Outcomes and complications

\begin{tabular}{lc}
\hline Characteristics & Included patients $(\mathrm{n}=18)$ \\
\hline ICU intensive treatment, $\mathrm{n}(\%)$ & $0(0.00)$ \\
Mortality during hospitalization, $\mathrm{n}(\%)$ & $0(0.00)$ \\
Oral feeding post-operation (days), median [IQR] & $3[2-20]$ \\
Local complications, $\mathrm{n}(\%)$ & $5(27.78)$ \\
Obvious abdominal symptom relief (days), median [IQR] & $1.5[1-2]$ \\
Additional intervention, $\mathrm{n}(\%)$ & $2(11.11)$ \\
Hospitalization time (days), median [IQR] & $5[3.75-9]$ \\
Hospitalization cost (RMB), median [IQR] & $25123.82[22,942.50-43,874.68]$ \\
Recurrence, $\mathrm{n}(\%)$ & $4(22.22)$ \\
\hline
\end{tabular}

ICU, intensive care unit; IQR, interquartile range.

Table 3 Pre- and 24 hours post-operative WBC and LIP levels of included patients

\begin{tabular}{lccc}
\hline & Pre-operation $($ mean \pm SD) & 24 hours post-operation $($ mean \pm SD) & P \\
\hline WBC $\left(\times 10^{9} / \mathrm{L}\right)$ & $14.57 \pm 7.15$ & $10.48 \pm 3.03$ & 0.007 \\
LIP $(\mathrm{U} / \mathrm{L})$ & $3,763.47 \pm 3,468.36$ & $1,438.76 \pm 1,490.09$ & 0.002 \\
\hline
\end{tabular}

WBC, white blood cells; LIP, lipase; SD, standard deviation.

Table 4 Pre- and 24 hours post-operative APACHE II and AMY levels of included patients

\begin{tabular}{lccc} 
& Pre-operation (median/quartile) & 24 hours post-operation (median/quartile) & $P$ \\
\hline APACHE II & $7.00(5.00-8.00)$ & $4.00(3.00-6.50)$ & 0.000265 \\
AMY (U/L) & $295.65(195.85-1,037.98)$ & $186.20(122.78-460.53)$ & 0.020
\end{tabular}

APACHE II, Acute Physiology and Chronic Health Evaluation II; AMY, Amylase.

The postoperative 24-hour leukocyte level, APACHE II score, serum amylase levels, and lipase levels significantly decreased compared with those at admission (Tables 3,4). All patients were discharged from hospital upon recovery, with a median hospitalization time of 5 (3.75-9) days (Figure 3). Stents were removed at 2-3 months after discharge. No endoscopic operation-related complications were found. None of the patients needed ICU treatment. Fifteen patients $(83.3 \%)$ achieved obvious abdominal pain relief and resumed oral feeding within 3 days. Two patients received additional surgical intervention for draining necrotic tissue. The median hospitalization cost was $25,123.82(22,942.50-43,874.68)$ RMB. Most of the patients were found to have pancreas divisum, and the MRCP images of 1 patient are shown in Figure 4.

\section{Follow up and long-term outcomes}

The median follow up time was 21.32 months (6-24 months). During the follow up, 4 patients $(22.22 \%)$ had AP recurrence (Table 2). One patient received conservative treatment in the local hospital and recovered afterwards. Two patients had recurrence after stent removal and received replacement of the stent through the minor papilla in our hospital. The other patient with hyperlipidemia had multiple recurrences when carrying stents. During the process of stent replacement, obstructions by thrombi were found.

\section{Discussion}

Currently, there are few reports regarding early endoscopic 

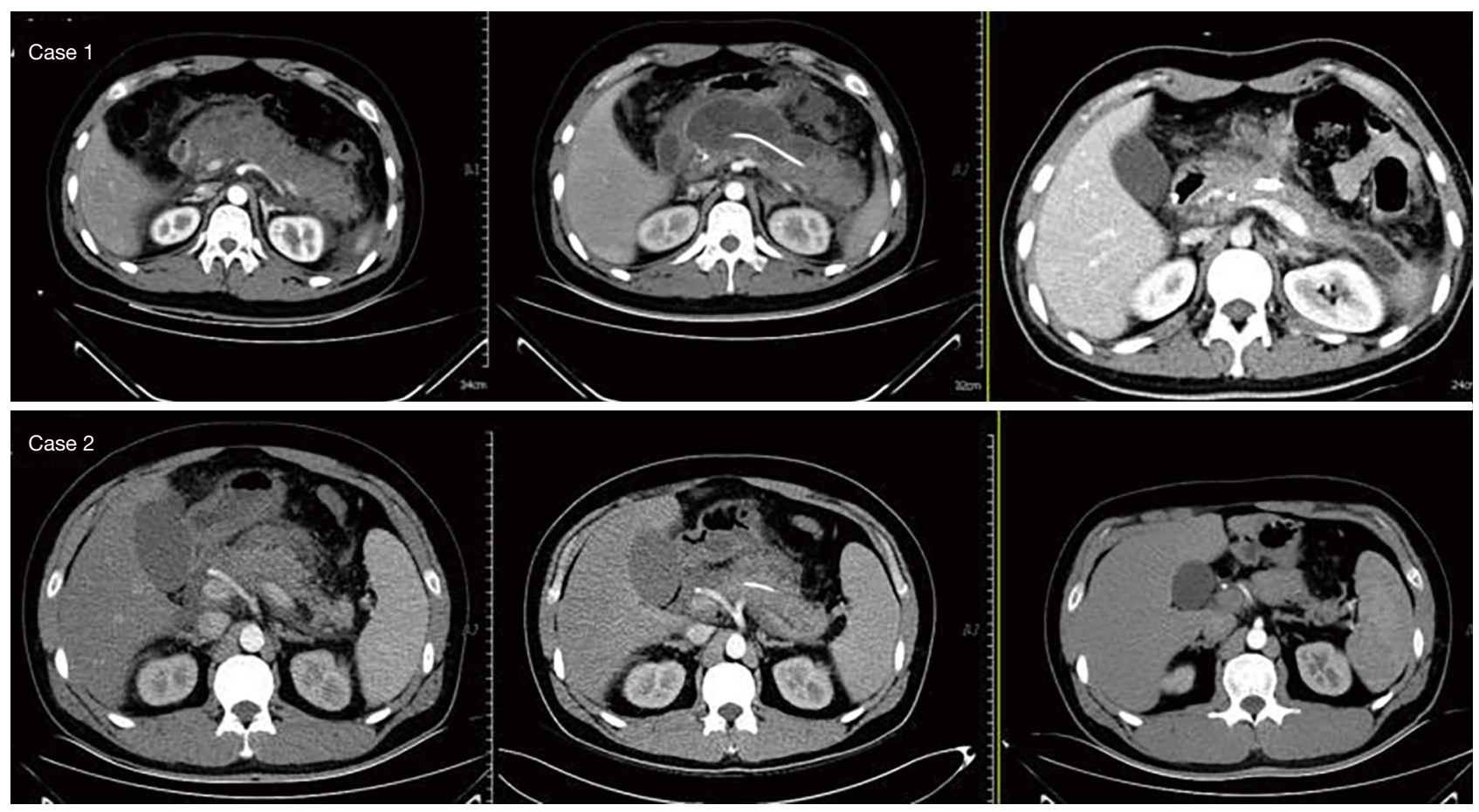

Figure 3 Pancreas recovery of 2 patients by pre-operation, 2 weeks after the operation, and 2 months after the operation.
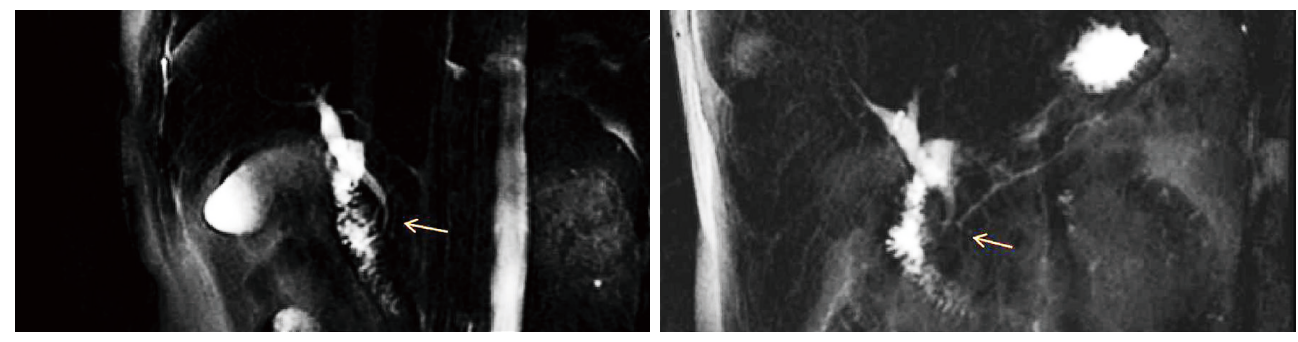

Figure 4 The minor pancreatic duct crossed the whole pancreas and entered the duodenum via the minor papilla as indicated by MRCP. The location indicated by the arrow indicates the site of pancreatic division. MRCP, magnetic resonance cholangiopancreatography.

transpapillary drainage through the minor papilla in the treatment of AP. In this report, we retrospectively evaluated the efficacy and safety of this strategy and technique among 18 patients treated in our hospital.

In the present study, all patients were discharged upon recovery and stents were removed at $2-3$ months after discharge. There were no endoscopic operation-related complications, and no ICU treatment was needed. Fifteen patients achieved abdominal pain relief and resumed oral feeding in 3 days. Two patients received additional surgical intervention for drainage of necrotic tissue. The median hospitalization time was 5 (3.75-9) days. According to previous reports of endoscopic treatment of AP, the mean pain relief time mainly ranged from 3-23.24 days $(16,21)$, the mean hospital stay ranged from 9.5-30.13 days $(10,16,21)$, the endoscopic operation-related complication rate ranged from $0-9 \%(21-23)$, and the morality rate ranged from $0-8 \%$ (22). The time to pain relief and hospital stay length were relatively short and the endoscopic operation-related complication rate was low in our study. Therefore, we can conclude that the strategy of early release of pancreatic duct obstruction and hypertension was feasible, and endoscopic transpapillary drainage through the minor papilla is safe and effective for AP.

Many studies have indicated that releasing pancreatic duct hypertension and obstruction is essential in AP 
treatment. Pancreatic duct obstruction and hypertension are universally present in AP regardless of its etiology $(8-12,24)$. The obstruction time of the pancreatic duct is closely associated with AP severity and prognosis, and early removal of the pancreatic duct obstruction can benefit AP remission, improve efficacy, and reduce the complication and mortality rate, as indicated by previous studies $(13-15,25,26)$. In contrast, if the pancreatic and biliary duct obstruction is not released early, pancreatitis progression can lead to edema of the duodenum and papilla, which can aggravate duct obstruction and lead to disease persistence and delayed recovery. At this time, the difficulty and risk of endoscopic treatment would greatly increase, and surgery would be necessary for drainage (16). In our study, during the endoscopic operation process, after cannulation via the minor papilla and subsequent suction, the obstruction mass was observed to flow out. This finding supports the hypothesis of pancreatic duct obstruction and hypertension and the importance of keeping the pancreatic duct under normal pressure during the acute phase.

Furthermore, most of the patients were identified to have pancreatic duct malformation in postoperative examinations. This indicated that the strategy of early release of the pancreatic duct obstruction and hypertension is also suitable for AP patients with pancreatic duct malformation, which is also one cause of AP. In these patients, anatomical differences may be found during the endoscopic operation: cannulation through the major pancreatic duct might be hard to perform, the minor papilla presents swelling and protrusion, or minor papilla cannulation may be easy to perform. Therefore, perioperative diagnostic techniques for pancreatic duct malformation such as ERCP would not be necessary, and physicians can rely on the actual finding during the endoscopic operation to make a decision on minor pancreatic duct drainage.

In the present study, we employed certain methods to reduce complications. Utilization of pancreatic duct stents can release pancreatic duct hypertension, prevent interaction of the enzyme and substrate, and prevent further aggravation after the operation. We did not perform regular oddi sphincterotomy and pancreatic duct sphincterotomy. In cases where sphincterotomy was necessary, we only performed small incision combined with papillary large balloon dilation, thus sphincter function could be retained and the complication risk could be reduced (27). ERCP itself may be associated with multiple complications, such as PEP, bleeding, digestive tract perforation, and fever, among others (28). The included patients were at the acute phase of pancreatitis, so we did not perform regular pancreatography in order to avoid pancreatitis aggravation. New technique detail was that trypsin drugs such as somatostatin and octreotide were not administered to patients, and the postoperative serum amylase and lipase levels decreased significantly.

In our study, minor papilla cannulation was successfully performed among all 18 included patients. According to a few previous studies, the success rate of cannulation via the minor papilla is usually low. Cotton et al. reported a success rate of $51.4 \%$ (29) and the rate obtained in the study by Yao et al. was $73 \%$ (30). The difficulty of minor papilla cannulation may be attributed to the anatomical features of the minor papilla. The minor papilla is located at the start of the descending duodenum, where it is difficult for a duodenoscope to steadily stay and easily crosses to the duodenal bulbar. Moreover, the minor papilla is small in size and requires patience and time to identify its position. In our study, we used a papillotome (Olympus KDV411M-720) and guidewire (COOK ACRO-35-450) for cannulation. The details of the operation are as follows: after identification of the minor papilla position, the visual field was optimized, the guidewire was extended to $2-3 \mathrm{~mm}$ above the cutter head, and sideling pushed the cutter head to the papilla; the cutter head was adjusted to be vertical to the papilla and the guidewire was used for exploration. In this technique, the patients are under steady anesthesia and breathing, and the operating physician is steady with a small movement range, thereby increasing the success rate.

However, there are still some limitations in the present study. Firstly, the sample size of the study was small. Secondly, the follow up duration was short, with some patients still in the follow up period. Thirdly, this is a retrospective and single arm study, which would lead to a certain bias. Further investigations are therefore warranted.

\section{Conclusions}

For AP patients, the strategy of early endoscopic release of pancreatic duct hypertension and obstruction is feasible, safe, and effective. It is also suitable for patients with pancreatic duct malformation when transpapillary drainage via the minor papilla can be performed.

\section{Acknowledgments}

Funding: This work was supported by the Key Research and Development Program of Ningxia Autonomous Region 
(No. 2020BEG02002), Ningxia Autonomous Region Science and technology benefiting the people program (No. $2021 \mathrm{cmg} 03013$ ) and the open subject of the application of pancreatic juice bacterial culture and the determination of pancreatic secretion miRNA in acute pancreatitisthe of General Hospital of Ningxia Medical University (clinical medical research center of autonomous region).

\section{Footnote}

Reporting Checklist: The authors have completed the STROBE reporting checklist. Available at https:// gs.amegroups.com/article/view/10.21037/gs-22-38/rc

Data Sharing Statement: Available at https://gs.amegroups. com/article/view/10.21037/gs-22-38/dss

Conflicts of Interest: All authors have completed the ICMJE uniform disclosure form (available at https://gs.amegroups. com/article/view/10.21037/gs-22-38/coif). The authors have no conflicts of interest to declare.

Ethical Statement: The authors are accountable for all aspects of the work in ensuring that questions related to the accuracy or integrity of any part of the work are appropriately investigated and resolved. All procedures performed in this study involving human participants were in accordance with the Declaration of Helsinki (as revised in 2013). The study was approved by institutional ethics board of General Hospital of Ningxia Medical University (No. KYLL-2022-1104) and informed consent was taken from all the patients.

Open Access Statement: This is an Open Access article distributed in accordance with the Creative Commons Attribution-NonCommercial-NoDerivs 4.0 International License (CC BY-NC-ND 4.0), which permits the noncommercial replication and distribution of the article with the strict proviso that no changes or edits are made and the original work is properly cited (including links to both the formal publication through the relevant DOI and the license). See: https://creativecommons.org/licenses/by-nc-nd/4.0/.

\section{References}

1. Peery AF, Dellon ES, Lund J, et al. Burden of gastrointestinal disease in the United States: 2012 update. Gastroenterology 2012;143:1179-87.e3.
2. Yu XN. Risk and severity prediction of severe acute pancreatitis. Chinese Journal of Gastroenterology and Hepatology 2015;24:234-7.

3. Heinrich S, Schäfer M, Rousson V, et al. Evidence-based treatment of acute pancreatitis: a look at established paradigms. Ann Surg 2006;243:154-68.

4. van Brunschot S, van Grinsven J, van Santvoort HC, et al. Endoscopic or surgical step-up approach for infected necrotising pancreatitis: a multicentre randomised trial. Lancet 2018;391:51-8.

5. Sun B and Li L, Update and progress for the diagnosis and treatment concept of severe acute pancreatitis. Chinese Journal of Practical Surgery 2014;34:235-9.

6. Yokoe M, Takada T, Mayumi T, et al. Japanese guidelines for the management of acute pancreatitis: Japanese Guidelines 2015. J Hepatobiliary Pancreat Sci 2015;22:405-32.

7. Pancreas Study Group, Chinese Society of Gastroenterology, Chinese Medical Association, et al. Chinese guidelines for the management of acute pancreatitis (Shanghai, 2013). J Clin Hepatol 2013;29:656-60.

8. Harvey MH, Wedgwood KR, Austin JA, et al. Pancreatic duct pressure, duct permeability and acute pancreatitis. $\mathrm{Br}$ J Surg 1989;76:859-62.

9. Neoptolemos JP, London NJ, Carr-Locke DL. Assessment of main pancreatic duct integrity by endoscopic retrograde pancreatography in patients with acute pancreatitis. $\mathrm{Br} \mathrm{J}$ Surg 1993;80:94-9.

10. Uomo G, Molino D, Visconti M, et al. The incidence of main pancreatic duct disruption in severe biliary pancreatitis. Am J Surg 1998;176:49-52.

11. Lau ST, Simchuk EJ, Kozarek RA, et al. A pancreatic ductal leak should be sought to direct treatment in patients with acute pancreatitis. Am J Surg 2001;181:411-5.

12. Shi CX, Chen JW, Carati CJ, et al. Effects of acute pancreatic duct obstruction on pancreatic perfusion: implication of acute pancreatic duct decompression. Scand J Gastroenterol 2002;37:1328-33.

13. Rosseland AR, Solhaug JH. Early or delayed endoscopic papillotomy (EPT) in gallstone pancreatitis. Ann Surg 1984;199:165-7.

14. Rünzi M, Saluja A, Lerch MM, et al. Early ductal decompression prevents the progression of biliary pancreatitis: an experimental study in the opossum. Gastroenterology 1993;105:157-64.

15. Kueppers PM, Russell DH, Moody FG. Reversibility of pancreatitis after temporary pancreaticobiliary duct 
obstruction in rats. Pancreas 1993;8:632-7.

16. Liao Z, Zheng L, Xie L. Fast treatment of acute pancreatitis duodenoscope (45 cases report). Sichuan Medical Journal 2011;32:313-4.

17. Ferri V, Vicente E, Quijano Y, et al. Diagnosis and treatment of pancreas divisum: A literature review. Hepatobiliary Pancreat Dis Int 2019;18:332-6.

18. Wang D, Dang R, Chen E, et al. Applied anatomy of duodenal papilla. Journal of Anatomy 2006;(6):781-3.

19. Delhaye M, Matos C, Devière J. Endoscopic technique for the management of pancreatitis and its complications. Best Pract Res Clin Gastroenterol 2004;18:155-81.

20. Raghuwanshi S, Gupta R, Vyas MM, et al. CT Evaluation of Acute Pancreatitis and its Prognostic Correlation with CT Severity Index. J Clin Diagn Res 2016;10:TC06-11.

21. Mo C, Sun G, Zhang L, et al. Early ERCP vs early conservative management in patients with severe acute biliary pancreatitis: A systematic review and Meta-analysis. World Chinese Journal of Digestology 2015;23:813-22.

22. Tian FZ, Zhang BY, Li DX, et al Effects of endoscopic biliary decompression on acute pancreatitis: a review of 20 years' experience. Chinese Journal of Practical Surgery 2005 ;(6):347-9.

23. Petrov MS, Uchugina AF, Kukosh MV. Does endoscopic retrograde cholangiopancreatography reduce the risk

Cite this article as: Yang Y, Yao W, Lan Z, Zhao C, Peng B, Dong T, Shi J, Wang Z. Early endoscopic transpapillary drainage through the minor papilla in the treatment of acute pancreatitis. Gland Surg 2022;11(2):442-450. doi: 10.21037/gs$22-38$ of local pancreatic complications in acute pancreatitis? A systematic review and meta-analysis. Surg Endosc 2008;22:2338-43.

24. Lerch MM, Saluja AK, Rünzi M, et al. Pancreatic duct obstruction triggers acute necrotizing pancreatitis in the opossum. Gastroenterology 1993;104:853-61.

25. Acosta JM, Rossi R, Galli OM, et al. Early surgery for acute gallstone pancreatitis: evaluation of a systematic approach. Surgery 1978;83:367-70.

26. Ricci F, Castaldini G, de Manzoni G, et al. Treatment of gallstone pancreatitis: six-year experience in a single center. World J Surg 2002;26:85-90.

27. Song J, Zhong Y, Lu X, et al. Enteral nutrition provided within 48 hours after admission in severe acute pancreatitis: A systematic review and meta-analysis. Medicine (Baltimore) 2018;97:e11871.

28. Chinese guidelines for ERCP (2018). Chinese Journal of Internal Medicine 2018;57:772-801.

29. Cotton PB. Congenital anomaly of pancreas divisum as cause of obstructive pain and pancreatitis. Gut 1980;21:105-14.

30. Yao W, Huang YH, Chang H, et al. Application Value of Duodenal Minor Papilla Cannulation in ERCP. Chinese Journal of Minimally Invasive Surgery 2016;16:889$891+895$. 